\title{
NOTES OF THE INVERSION OF INTEGRALS I
}

\author{
GEORGE R. KEMPF
}

(Communicated by Jonathan M. Rosenberg)

\begin{abstract}
If $W$ is a Picard bundle on the Jacobian $J$ of a curve $C$, we have the problem of describing $W$ globally. The theta divisor $\theta$ is ample on $J$. Thus it is possible to write $n^{*} W$ as the sheaf associated to a graded $M$ over the well-known ring $\oplus_{m>0} \Gamma\left(J, \mathscr{C}_{J}(m 4 \theta)\right)$. In this paper we compute the degree of generators and relations for such a module $M$.
\end{abstract}

There are naturally occurring locally free sheaves called Picard bundles on the Jacobian $J$ of a smooth complete curve $C$ of positive genus $g$ over $k=\bar{k}$. These bundles describe the global variation of the sections of invertible sheaves on $C$ with pleasant degree.

The inversion problem is to give a description of the Picard bundles globally on $J$. As such analytic description is lacking, we must content ourselves with two algebraic solutions of this problem.

The first solution requires us to know the image of some points of $C$ in the Jacobian. This approach uses a method due to R. C. Gunning. The second solution determines the pull-back of the Picard bundle by a multiplication in $J$ in terms of a module over the graded ring of theta sections. Here one uses a form of a theorem of $\mathrm{D}$. Mumford on the equations defining abelian varieties projectively.

\section{THE FIRST METHOD}

Let $\mathscr{P}$ be a Poincaré sheaf on $J \times J$. Let $\mathscr{L}_{n}$ be an invertible sheaf on $J$ of the form $\mathscr{O}_{j}(n \theta)$ where the divisor $\theta$ gives the usual principal polarization of $J$. If $n>0$ then $\pi_{2}^{*} \mathscr{L}_{n} \otimes \mathscr{P}$ is a family of ample invertible sheaves on the second factor. It follows from Mumford's vanishing theorem that

$$
R^{i} \pi_{1^{*}}\left(\pi_{2}^{*} \mathscr{L}_{n} \otimes \mathscr{P}\right)
$$

is zero if $i>0$ and $\mathscr{V}_{n}=\pi_{1 \cdot}\left(\pi_{2}^{*} \mathscr{L}_{n} \otimes \mathscr{P}\right)$ is a locally free sheaf of rank $n^{g}$.

Let $C \hookrightarrow J$ be a universal abelian integral. Let $Q_{n}=\left.\pi_{2}^{*} \mathscr{L}_{n} \otimes \mathscr{P}\right|_{J \times C}$. Then $Q_{n}$ is a family of invertible sheaves on $C$ of degree $n \cdot g$ as $[C: \theta]=g$. If

Received by the editors November 17, 1988.

1980 Mathematics Subject Classification (1985 Revision). Primary 14H40; Secondary 14K20, $14 \mathrm{~K} 25$.

Key' words and phrases. Algebraic curves, Jacobians, Picard bundles. 
$n>1$ then $R^{i} \pi_{J^{*}} Q^{n}$ is zero if $i>0$ and the Picard sheaf $\mathscr{W}_{n g}=\pi_{J^{*}} Q_{n}$ is locally free of rank $n g-g+1=(n-1) g+1$.

The inclusion of $C$ in $J$ induces a restriction homomorphism $\alpha: \mathscr{U}_{n} \rightarrow$ $\mathscr{W}_{n g}$. The critical fact is

Proposition 1 (Gunning [2]). $\alpha$ is surjective if $n>1$.

Let $D$ be an effective divisor on $C$ of degree $d$. Then $Q_{n}(-J \times D)$ is a family of invertible sheaves on $C$ of degree $n \cdot g-d$. If $d>n \cdot g$, then $\pi_{J^{*}}\left(Q_{n}(-J \times D)\right)$ is zero and the Picard sheaf $\mathscr{U}_{n}(D) \equiv R_{J^{*}}^{1}\left(Q_{n}(-J \times D)\right)$ is locally free of rank $d-n \cdot g+g-1=d-(n-1) g-1$. Consider the exact sequence

$$
\left.0 \rightarrow Q_{n}(-J \times D) \hookrightarrow Q_{n} \rightarrow Q_{n}\right|_{J \times D} \rightarrow 0 .
$$

This yields the well-known exact sequence of

Lemma 2. We have an exact sequence

$$
0 \rightarrow \mathscr{W}_{n g} \stackrel{e}{\longrightarrow} \pi_{J^{*}}\left(\left.Q_{n}\right|_{J \times D}\right) \rightarrow \mathscr{U}_{n}(D) \rightarrow 0
$$

where $e$ is just evaluation.

The composition $\beta_{D}: \mathscr{V}_{n} \stackrel{\leftrightarrow}{\longrightarrow} \mathscr{W}_{n g} \stackrel{e}{\longrightarrow} \pi_{J^{*}}\left(\left.Q_{n}\right|_{J \times D}\right)=\pi_{J^{*}}\left(\left.\pi_{2}^{*} \mathscr{L}_{n} \otimes \mathscr{P}\right|_{J \times D}\right)$ is simply restriction and is determined only by how $D$ sits as a closed subscheme of $J$. The combination of the above facts give the first solution of the inversion problem.

Theorem 3. $\mathscr{W}_{n g}=\operatorname{Image}\left(\beta_{D}\right)$ and $\mathscr{U}_{n}(D)=\operatorname{Cokernel}\left(\beta_{D}\right)$.

\section{NORMAL PRESENTATION}

Let $\mathscr{L}$ be a very ample sheaf on a projective variety $X$. A coherent sheaf $\mathscr{F}$ on $X$ is said to be normally presented if we have an exact sequence

$$
R \otimes_{k} \mathscr{L}^{\otimes-1} \stackrel{\alpha}{\longrightarrow} G \otimes_{k} \mathscr{O}_{X} \rightarrow \mathscr{F} \rightarrow 0
$$

for some vector spaces $R$ and $G$. Furthermore $\mathscr{F}$ is said to be strongly presented if the homomorphism $G \rightarrow \Gamma(X, \mathscr{F})$ is surjective.

Lemma 4. A strongly presented coherent sheaf $\mathscr{F}$ is determined by $\Gamma(X, \mathscr{F})$ and the kernel of the multiplication

$$
\Gamma(X, \mathscr{F}) \otimes \Gamma(X, \mathscr{L}) \rightarrow \Gamma(X, \mathscr{F} \otimes \mathscr{L}) .
$$

Proof. First of all we may assume that $\beta: G \rightarrow \Gamma(X, \mathscr{F})$ is an isomorphism by factoring $G \otimes_{k} \mathscr{O}_{X} \rightarrow \mathscr{F}$ through $\bar{G} \otimes_{k} \mathscr{O}_{X}$ where $\bar{G}$ is the image of $\beta$. Then $R \rightarrow G \otimes \Gamma(X, \mathscr{L})$ has image in the kernel of multiplication. Hence the kernel contains enough relations to define $\mathscr{F}$ as a quotient sheaf of $G \otimes_{k} \mathscr{O}_{X}$.

We will need a lemma to prove that some sheaves are strongly presented. 
Lemma 5. Given an exact sequence

$$
0 \rightarrow \mathscr{F}_{1} \rightarrow \mathscr{F}_{2} \rightarrow \mathscr{F}_{3} \rightarrow 0
$$

of coherent sheaves on $X$, assume that

(a) $\mathscr{F}_{2}$ is strongly normally presented, and

(b) $\mathscr{F}_{1}$ is generated by its sections and $H^{1}\left(X, \mathscr{F}_{1}\right)$ is zero. Then $\mathscr{F}_{3}$ is strongly normally presented.

Proof. By (b) we have an exact sequence, $0 \rightarrow \Gamma\left(X, \mathscr{F}_{1}\right) \rightarrow \Gamma\left(X, \mathscr{F}_{2}\right) \rightarrow$ $\Gamma\left(X, \mathscr{F}_{3}\right) \rightarrow 0$ and a surjection $\Gamma\left(X, \mathscr{F}_{1}\right) \otimes_{k} \mathscr{F}_{X} \rightarrow \mathscr{F}_{1}$. By (a) we have an exact sequence

$$
R \otimes_{k} \mathscr{L}^{\otimes-1} \rightarrow \Gamma\left(X, \mathscr{F}_{2}\right) \otimes \mathscr{O}_{X} \rightarrow \mathscr{F}_{2} \rightarrow 0
$$

(using the proof of Lemma 4). Therefore we have an exact sequence

$$
R \otimes_{k} \mathscr{L}^{\otimes-1} \oplus \Gamma\left(X, \mathscr{F}_{1}\right) \otimes_{k} \mathscr{O}_{X} \rightarrow \Gamma\left(X, \mathscr{F}_{2}\right) \otimes \mathscr{O}_{X} \rightarrow \mathscr{F}_{3} \rightarrow 0
$$

which we can factor as

$$
R \otimes_{k} \mathscr{L}^{\otimes-1} \rightarrow \Gamma\left(X, \mathscr{F}_{3}\right) \otimes \mathscr{O}_{X} \rightarrow \mathscr{F}_{3} \rightarrow 0
$$

Remark. If we just assume that $\mathscr{F}_{1}$ is generated by its sections, then we can conclude that $\mathscr{F}_{3}$ is normally presented.

\section{Abelian varieties}

Let $X$ be an abelian variety with ample invertible sheaf $\mathscr{L}$. An invertible sheaf $\mathscr{M}$ on $X$ is said to be of type $n$ if it is algebraically equivalent to $\mathscr{L}^{\otimes n}$ for some integer $n$. If the type is $\mathscr{M} \geq 1$ then $\mathscr{M}$ is ample and if it is $\geq 2$ then $\mathscr{M}$ is generated by its sections and if it is $\geq 3$ then $\mathscr{M}$ is very ample.

We have a basic result.

Theorem 6. If $\mathscr{N}$ and $\mathscr{M}$ are two invertible sheaves on the abelian variety $X$ such that type $(\mathscr{N}) \geq 3$ and type $(\mathscr{M}) \geq 4$, then $\mathscr{N}$ is strongly normally generated for $\mathscr{M}$.

Proof. We first need to write enough relations between the sections of $\mathscr{N}$ and $\mathscr{M}$. Let $Q_{\alpha}$ be an invertible sheaf of type 2 . We may write $\mathscr{N}=\mathscr{R}_{\alpha} \otimes Q_{\alpha}$ and $\mathscr{M}=\mathscr{S}_{\alpha} \otimes Q_{\alpha}$ where type $\left(\mathscr{R}_{\alpha}\right) \geq 1$ and type $\left(\mathscr{S}_{\alpha}\right) \geq 2$. Let $r \in \Gamma\left(X, \mathscr{R}_{\alpha}\right)$, $s \in \Gamma\left(X, \mathscr{S}_{\alpha}\right)$ and $q_{1}$ and $q_{2} \in \Gamma\left(X, \mathscr{Q}_{\alpha}\right)$. Let $\langle$,$\rangle denote the product of two$ sections. Evidently

$$
a\left(r, s, q_{1}, q_{2}\right)=\left\langle r, q_{1}\right\rangle \otimes\left\langle s, q_{w}\right\rangle-\left\langle r, q_{2}\right\rangle \otimes\left\langle s, q_{1}\right\rangle
$$

is contained in the kernel of the multiplication

$$
\Gamma(X, \mathscr{N}) \otimes \Gamma(X, \mathscr{M}) \rightarrow \Gamma(X, \mathscr{N} \otimes \mathscr{M}) .
$$

Let $A$ be the span of all possible such relations $a\left(r, s, q_{1}, q_{2}\right)$ for all possible $\alpha$. 
Let $N=\Gamma(X, \mathscr{N}) \otimes_{k} B / A B$ where $B$ is the graded ring $\bigoplus_{n \geq 0} \Gamma\left(X, \mathscr{M}^{\otimes n}\right)$. We have a canonical surjection $\pi: \widetilde{N} \rightarrow \mathscr{N}$ where $\widetilde{N}$ is the $\mathscr{O}_{X}$-module associated to the $A$-module $N$. The theorem will be proven if we can show that $\pi$ is an isomorphism.

To do the above we must show that (1) for any point $x$ of $X$ the vector space $\widetilde{N}(x)$ is one-dimensional.

We may assume that all sheaves on $X$ have been given compatible trivialization at $x$ and let $e(\sigma)=\sigma(x)$ be evaluation. Then $\widetilde{N}(x)=\Gamma(X, \mathcal{N}) /(1 \otimes e) A$ by definition. Thus we need to show that $(1 \otimes e) A$ has codimension one in $\Gamma(X, \mathcal{N})$. As the whole theorem is invariant under translation we may assume that $x$ is the identity 0 of $X$.

Take $\lambda: \Gamma(X, \mathcal{N}) \rightarrow k$ a linear functional such that $\lambda((1 \otimes e) A)=0$. Now $(1 \otimes e) a\left(r, s, q_{1}, q_{1}\right)=(1 \otimes s(0))\left(\langle r, q\rangle q_{2}(0)-\left\langle r, q_{2}\right\rangle q_{1}(0)\right.$. As $S_{\alpha r}$ is generated by its sections we may assume that $s(0) \neq 0$. Therefore $\lambda\left(\left\langle r, q_{1}\right\rangle q_{2}(0)\right)=$ $\lambda\left(\left\langle r, q_{2}\right\rangle q_{1}(0)\right)$ is symmetric in $q_{1}$ and $q_{2}$ and vanishes if $q_{1}(0)$ or $q_{2}(0)$ equals zero. Write $\lambda\left(\left\langle r, q_{1}\right\rangle q_{2}(0)\right)=\mu(r) q_{1}(0) q_{2}(0)$ and note that $\mu$ is well defined because $Q_{r r}$ is generated by its sections. We intend to show that (2) $\mu_{r r}(r)=$ constant $\cdot r(0)$. If we show (2) then $\lambda$ is a multiple of evaluation at 0 . Hence $(1 \otimes e) A$ is the kernel of evaluation and thus (1) is true.

We will show that (2) follows from a global variational argument with $\alpha$. Let $\mathscr{P}$ be a Poincare sheaf on $X \otimes \widehat{X}$ where $\widehat{X}$ is the dual abelian variety. Let $\mathscr{R}$ be one possible choice of $\mathscr{R}_{\alpha}$. Then all possible choices are the restriction of $\pi_{X}^{*} \mathscr{R} \otimes \mathscr{P}$ to the fibers of $\pi_{\hat{X}}$. Globally $\mu_{k \alpha}$ is the value of a $\mathscr{O}_{\widehat{X}}$-homomorphism $\mu: \mathscr{W} \equiv \pi_{\widehat{X}}\left(\pi_{X}^{*} \mathscr{R} \otimes \mathscr{P}\right) \rightarrow \mathscr{O}_{X}$. By [1,4] $H^{\operatorname{dim} \hat{X}}(\widehat{X}, \mathscr{W})$ is onedimensional. Hence by duality $\operatorname{Hom}_{\widehat{X}}\left(\mathscr{W}, \mathscr{O}_{\widehat{X}}\right)$ is one-dimensional but evaluation at 0 is one such homomorphism. Hence $\mu$ is a multiple of evaluation. Therefore (2) is true.

When $\mathscr{N}=\mathscr{M}$ the theorem follows from D. Mumford's theorem $[3,4]$ that $\bigoplus_{k \geq 0} \Gamma\left(X, \mathscr{M}^{\otimes k}\right)$ is almost normally presented as a ring. The proof of the theorem is close to Mumford's reasoning but the technicalities are easier.

\section{THE SECOND METHOD}

An invertible sheaf $\mathscr{L}$ on the Jacobian $J$ has type $n$ if $\mathscr{L}$ is algebraically equivalent to $\mathscr{O}_{J}(n \theta)$ where $\theta$ is in the class of the principal polarization. Thus $\operatorname{type}\left(\mathscr{L}_{n}\right)=n$ where $\mathscr{L}_{n}$ is the sheaf of $\S 1$, the notation of which we will be using.

Let $\mathscr{M}(\mathscr{R})$ be invertible sheaves on $J$ of type $m(r)$. One might hope to prove that $\mathscr{U}_{n}(D) \otimes \mathscr{M}$ is normally presented for $\mathscr{R}$ for reasonable bounds on $m, n$ and $r$. If one tries to use Lemma 2 and Lemma 5, the problem is that we would need $\mathscr{W}_{n g} \otimes \mathscr{M}$ to be generated by its sections (but I do not know when this is true). This emphasis is circumvented by applying the isogeny 
$n L_{J}: J \rightarrow J$ given by multiplication by $n$. This resolves the problem. The result is

Theorem 7. (a) $\left(\left(n 1_{J}\right)^{*} \mathscr{U}_{n}(D)\right) \otimes \mathscr{M}$ is normally presented for $\mathscr{R}$ if $m \geq$ $n+2 \geq 4$ and $r \geq 4$.

(b) It is strongly normally presented for $\mathscr{R}$ if $m \geq n+2 \geq 4, r \geq 4$ and, if $n \geq 3$, then $g \geq 2$ and $m /(m, n)$ prime to $\operatorname{char}(k)$.

Proof. First of all we may assume that the effective divisor $D$ consists of distinct point $e_{1}, \ldots, e_{d}$. This follows because the isomorphism class of $\mathscr{U}_{n}(D)$ only depends on that of $\left.\mathscr{L}_{n}\right|_{C} ^{(D)} \equiv \mathscr{H}$ but we may vary $\mathscr{L}_{n}$ and $D$ so that $D$ is reduced while not changing $\mathscr{H}$.

Then from Lemma 2 we have an exact sequence

$$
0 \rightarrow \mathscr{W}_{n g} \rightarrow \bigoplus_{1 \leq i \leq d} \mathscr{S}_{i} \rightarrow \mathscr{U}_{n}(D) \rightarrow 0
$$

where $\mathscr{S}_{i}=\pi_{J^{*}}\left(\left.Q_{n}\right|_{J \times \mathcal{C}_{i}}\right)$. Now type $\left(\mathscr{S}_{i}\right)=0$ because

$$
\left.Q_{n}\right|_{J \times e_{i}}=\left.\left(\pi_{1}^{*} \mathscr{L}_{n} \otimes \mathscr{P}\right)\right|_{J \times e_{i}}=\left.\mathscr{L}_{n}\left(e_{i}\right) \otimes_{k} \mathscr{P}\right|_{J \times e_{i}},
$$

which is algebraically equivalent to $\mathscr{O}_{J}$.

Next we pull this sequence back and get

(1) $0 \rightarrow\left(n 1_{J}\right)^{*} \mathscr{W}_{n g} \rightarrow \bigoplus_{l \leq i \leq d} \mathscr{T}_{i} \rightarrow\left(n \times 1_{J}\right)^{*} \mathscr{U}_{n}(D) \rightarrow 0$, where type $\left(\mathscr{T}_{i}\right)=$ 0 and $\mathscr{T}_{i}=\left(n 1_{J}\right)^{*} \mathscr{S}_{i}$. Now $\left(\bigoplus_{l<i<d} \mathscr{T}_{i}\right) \otimes \mathscr{M}$ is strongly normally presented for $\mathscr{R}$ if $m \geq 3$ and $r \geq 4$ by Theorem 6 . Thus point (a) will follow from (1) $\otimes \mathscr{M}$ and the remark after Lemma 5 if we can prove that

(2) $\left(n 1_{J}\right)^{*} \mathscr{W}_{n g} \otimes \mathscr{M}$ is generated by its sections if $m \geq n+2$. Also by Lemma 5 the point (b) will follow if we prove

(3) $H^{1}\left(J,\left(n 1_{J}\right)^{*} \mathscr{W}_{n g} \otimes \mathscr{M}\right)=0$ if $m \geq n+2 \geq 4$ and if $n \geq 3$ then $m>(n /(n-2))^{1 / 2 g-1} n$ and $m /(m, n)$ prime to $\operatorname{char}(k)$.

To prove (2) we will use the surjection $\alpha: \mathscr{V}_{n} \rightarrow \mathscr{W}_{n g}$ of Proposition 1 as $n \geq 2$. Thus (2) will follow if we prove

(4) $\left(n 1_{J}\right)^{*} \mathscr{V}_{n} \otimes \mathscr{M}$ is generated by its sections if $m \geq n+2$.

By [1] $\left(n 1_{J}\right)^{*} \mathscr{V}_{n} \approx \Gamma\left(J, \mathscr{L}_{n}\right) \otimes_{k} \mathscr{L}_{n}^{\otimes-1}$. Hence we just need $\left(\mathscr{L}_{n}^{\otimes-1} \otimes \mathscr{R}\right)$ to be generated by its sections; e.g. its type $\geq 2$. As the type is $m-n$, (4) is true.

To prove (3) we have to modify the argument of [1] due to the presence of $\left(n 1_{J}\right)^{*}$. We will first give some isomorphisms which follow in the same way as [1] from the vanishing of higher direct images and the Leray spectral sequence.

$$
H^{i}\left(J\left(n 1_{J}\right)^{*} \mathscr{W}_{n g} \otimes \mathscr{M} \simeq H^{j}\left(J \times C, \pi_{C}^{*} \mathscr{L}_{n} \otimes \mathscr{P}^{n} \otimes \pi_{J}^{*} \mathscr{M}_{J \times C}\right)\right.
$$


as

$$
\begin{aligned}
\left(n 1_{J}\right)^{*} \mathscr{W}_{n g} & =\pi_{J^{*}}\left(\left.\left(n 1_{J} \times 1_{C}\right)^{*}\left(\pi_{C}^{*} \mathscr{L}_{n} \otimes \mathscr{P}\right)\right|_{J \times C}\right) \\
& =\pi_{J^{*}}\left(\left.\pi_{C}^{*} \mathscr{L}_{n} \otimes \mathscr{P}^{\otimes n}\right|_{J \times C}\right) .
\end{aligned}
$$

$H^{i}\left(J \times C,\left.\pi_{C}^{*} \mathscr{L}_{n} \otimes \mathscr{P}^{n} \otimes \pi_{J}^{*} \mathscr{M}\right|_{J \times C)}=H^{i}\left(C, \mathscr{L}_{n} \otimes\left(n 1_{J}\right)^{*}\left(\pi_{2^{*}}\left(\mathscr{P} \otimes \pi_{1}^{*} \mathscr{M}\right)\right) \mid C\right)\right.$ as $\left.\left.\left.\pi_{C^{*}} \mathscr{L}_{n}\right|_{C} \otimes\left(\mathscr{P}^{n} \otimes \pi_{J}^{*} \mathscr{M}\right)\right|_{J \times C}\right)\left.\simeq \pi_{2^{*}}\left(\pi_{2}^{*} \mathscr{L}_{n} \otimes\left(1_{J} \times n 1_{J}\right)^{*}\left(\mathscr{P} \times \pi_{1}^{*} \mathscr{M}\right)\right)\right|_{C} \simeq$ $\left.\mathscr{L}_{n} \otimes\left(n 1_{J}\right)^{*}\left(\pi_{2^{*}}\left(\mathscr{P} \otimes \pi_{1}^{*} \mathscr{M}\right)\right)\right|_{C}$.

Thus

$$
H^{i}\left(J\left(n 1_{J}\right)^{*} \mathscr{W}_{n g} \otimes \mathscr{M}\right) \simeq H^{i}\left(C,\left.\mathscr{L}_{n} \otimes\left(n 1_{J}\right)^{*}\left(\pi_{2^{*}}\left(\mathscr{P} \otimes \pi_{1}^{*} \mathscr{M}\right)\right)\right|_{C}\right) .
$$

Now we need to determine when the cohomology of this sheaf on $C$ vanishes. Let $a=m /(m, n)$. Write $m=a b$ and $n=c b$. As $a$ is prime to the characteristic, the curve $C_{a}=\left(a 1_{J}\right)^{-1} C$ is an unramified Galois covering of $C$. Hence for any quasicoherent sheaf $\mathscr{F}$ on $C$ we have an injection $H^{i}(C \mathscr{F}) \hookrightarrow$ $H^{i}\left(C_{a},\left(a 1_{J}\right)^{*} \mathscr{F}\right)$. Thus we want to study

$$
\left.H^{1}\left(C_{a},\left(a 1_{J}\right)^{*}(\mathscr{L}) n \otimes\left(n 1_{J}\right)^{*}\left(\pi_{2^{*}}\left(\mathscr{P} \otimes \pi_{1}^{*} \mathscr{M}\right)\right)\right)_{C_{a}}\right) .
$$

Here

$$
\begin{aligned}
\left(a 1_{J}\right)^{*} & \left(\mathscr{L}_{n} \otimes\left(n 1_{J}\right)^{*}\left(\pi_{2^{*}}\left(\mathscr{P} \otimes \pi_{1}^{*} \mathscr{M}\right)\right)\right) \\
& \simeq\left(a 1_{J}\right)^{*} \mathscr{L}_{n} \otimes\left(c 1_{J}\right)^{*}\left(m 1_{J}\right)^{*}\left(\pi_{2^{*}}\left(\mathscr{P} \otimes \pi_{1}^{*} \mathscr{M}\right)\right) \\
& \simeq\left(a 1_{J}\right)^{*} \mathscr{L}_{n} \otimes\left(c 1_{J}\right)^{*} \mathscr{M}^{\otimes-1} \otimes_{k} \Gamma(J, \mathscr{M})
\end{aligned}
$$

as $\left(m 1_{J}\right)^{*}\left(\pi_{2^{*}}\left(\mathscr{P} \otimes \pi_{1}^{*} \mathscr{M}\right)\right) \simeq \mathscr{M}^{\otimes-1} \otimes_{k} \Gamma(J, \mathscr{M})$. Thus the first cohomology groups vanish if

$$
\begin{aligned}
\operatorname{deg}\left(\left(a 1_{J}\right)^{*} \mathscr{L}_{n} \otimes\left(c 1_{J}\right)^{*} \mathscr{M}^{\otimes-1} \dot{\mid}_{C_{a}}\right) & >2\left(\operatorname{genus}\left(C_{a}\right)-1\right) \\
& =2 \operatorname{deg}\left(a 1_{J}\right)(g-1) \\
& =2 a^{2 g}(g-1)
\end{aligned}
$$

but the degree of the sheaf is $a^{2 g} n g-c^{2 g} m g$. Finally for the vanishing we need the inequality $a^{2 g} g n-c^{2 g} g m>2 a^{2 g}(g-1)$ or rather $2 m^{2 g-1} / g n+m^{2 g-1}>$ $n^{2 g-1}+(2 / n) m^{2 g-1}$. For $n \geq 3$, this is true if $m>(n /(n-2))^{1 / 2 g-1} n$, or, if $n=2$ if $m>2 g^{1 / 2 g-1}$. The simplest case of part (b) is $n=3$ and $m=5$ if $g \geq 2$ or $n=2$ and $m=4$.

The theorem tells us when $\left(\left(n I_{J}\right)^{*} \mathscr{U}_{n}(D)\right) \otimes \mathscr{M}$ is determined by the multiplication

$$
\beta: \Gamma\left(J,\left(\left(n 1_{J}\right)^{*} \mathscr{U}_{n}(D)\right) \otimes \mathscr{M}\right) \otimes \Gamma(J, \mathscr{R}) \rightarrow \Gamma\left(J,\left(\left(n 1_{J}\right)^{*} \mathscr{U}_{n}(D)\right) \otimes \mathscr{M} \otimes \mathscr{R}\right) .
$$

Thus we want to know more about this group. I will only give the dimension here. 
Theorem 8. (a) $H^{i}\left(J,\left(\left(n 1_{J}\right)^{*} \mathscr{U}_{n}(D)\right) \otimes \mathscr{M}\right)=0$ if $i>0$ and $m \geq 1$.

(b) $\operatorname{dim} \Gamma\left(J,\left(\left(n 1_{J}\right)^{*} \mathscr{U}_{n}(D)\right) \otimes \mathscr{M}\right)=\left((d-g n+g-1) m^{g}+g n^{2} m^{g-1}\right)$ if $m \geq 1$.

Proof. Consider the long exact sequence of cohomology of the sequence (1) tensored with $\mathscr{M}$. If $m \geq 1$ then the higher cohomology groups of the sheaf $\mathscr{T}_{i} \otimes \mathscr{M}$ vanish and by the isomorphism $\circledast$ of the last proof as

$$
H^{i}\left(J,\left(n 1_{J}\right)^{*} \mathscr{W}_{n g} \otimes \mathscr{M}\right)=0 \quad \text { if } i \geq 1=\operatorname{dim} C .
$$

Thus (a) is true by the long exact sequence.

By (a) the dimension is the Euler characteristic $\chi\left(\left(n 1_{J}\right)^{*} \mathscr{U}_{n}(D) \otimes \mathscr{M}\right)$, which I intend to compute using the Hirzebruch-Riemann-Roch theorem. We need to know $\operatorname{ch}\left(\left(n 1_{J}\right)^{*} \mathscr{U}_{n}(D) \otimes \mathscr{M}\right)$ because its number of codimension $g$ cycles $(=$ points) is the Euler characteristic as the Todd class of $J$ is 1 .

By Mattuck's result $c_{t}\left(\mathscr{U}_{n}(D)\right)=\sum_{i \geq 0} w_{i} t^{i}$ is algebraic equivalent where $w_{i}$ is the image of $C^{(g-1)}$ in $J$. Thus by Poincaré relation, $c_{t}\left(\mathscr{U}_{n}(D)\right)=\exp (\theta t)$ in numerical equivalence. Now if $c_{t}\left(\mathscr{U}_{n}(\theta)\right)=\prod_{1 \leq i \leq n} 1+k_{i} t$, we get $+t \theta=$ $\log (\exp (\theta t))=+\sum_{i} \log \left(1+w_{i} t\right)=\sum_{p \geq 1} \sum_{i}(-1)^{p} w_{i}^{p t p} / p$ but $\operatorname{ch}_{t}\left(\mathscr{U}_{n}(D)\right)=$ $\sum_{i} \exp \left(w_{i} t\right)=\sum_{p} \sum_{i} w_{i}^{p} / p^{i}$. Comparing coefficients we find

$$
\operatorname{ch}_{t}\left(\mathscr{U}_{n}(D)\right)=\operatorname{rank}\left(\mathscr{U}_{n}(D)\right)+t c_{1}\left(\mathscr{U}_{n}(D)\right)=\operatorname{rank}+t \theta,
$$

where rank $=-g \cdot n+d+g=1$. Hence $\operatorname{ch}\left(\left(n 1_{J}\right)^{*} \mathscr{U}_{n}(D)\right)=\operatorname{rank}+n^{2} \theta$. Thus

$$
\operatorname{ch}\left(\left(n 1_{J}\right)^{*} \mathscr{U}_{n}(D) \otimes \mathscr{M}=\operatorname{ch}\left(\left(n 1_{J}\right)^{*} \mathscr{U}_{n}(D)\right) \cdot \operatorname{ch}(\mathscr{M})=\operatorname{rank}+n^{2} \theta\right) \exp (m \theta) .
$$

Therefore $\chi\left(\left(n 1_{J}\right)^{*} \mathscr{U}_{n}(D) \otimes \mathscr{M}\right)=($ rank $) m^{g}+n^{2} m^{g-1} g$ and the result follows.

\section{A last remark is}

Theorem 9. In the range of Theorem 7(b) then the multiplication $\beta$ is surjective. Proof. The conditions of Theorem 7 (b) are true when $\mathscr{M}=\mathscr{M} \otimes \mathscr{R}$. The proof shows that the homomorphism

$$
\Gamma\left(J, \oplus \mathscr{T}_{i} \otimes \mathscr{M}\right) \rightarrow \Gamma\left(J,\left(m 1_{J}\right)^{*} \mathscr{U}_{n}(D) \otimes \mathscr{M}\right)
$$

is surjective for $\mathscr{M}=\mathscr{M}$ and $\mathscr{M} \otimes \mathscr{R}$. This theorem results because the multiplication

$$
\Gamma\left(J, \mathscr{T}_{i} \otimes \mathscr{M}\right) \otimes \Gamma(J, \mathscr{R}) \rightarrow \Gamma\left(J, \mathscr{T}_{i} \otimes \mathscr{M} \otimes \mathscr{R}\right)
$$

is surjective by Mumford's result in [4].

\section{REFERENCES}

1. G. Kempf, Toward the inversion of abelian integrals II, Amer. J. Math. 101 (1979). 184-202.

2. __ Some metrics on Picard bundles, in "Vector Bundles on Algebraic Varieties," Tata Institute, Bombay, pp. 217-224. 
3. D. Mumford, On the equations defining abelian varieties I, II, III, Inventiones Math. 1 (1966) 287-354; 3 (1967) 75-135 and 215-244.

4. _. Varieties defined by quadratic equations, in "Questions on Algebraic Varieties," Centro Inter. Mate, Estrivo, Roma (1970), 31-100.

Department of Mathematics, Johns Hopkins University. Baltimore, Maryland 21218 